\title{
Does Flagging POTUS's Tweets Lead to Fewer or More Retweets? Preliminary Evidence from Machine Learning Models
}

\author{
Wallace Chipidza (corresponding author) \\ Claremont Graduate University \\ wallace.chipidza@cgu.edu
}

\author{
Jie (Kevin) Yan \\ Dalton State College \\ iyan@daltonstate.edu
}

\begin{abstract}
There is vigorous debate as to whether influential social media platforms like Twitter and Facebook should censor objectionable posts by government officials in the United States and elsewhere. Although these platforms have resisted pressure to censor such posts in the past, Twitter recently flagged five posts by the United States President Donald J. Trump on the rationale that the tweets contained inaccurate or inflammatory content. In this paper, we examine preliminary evidence as to whether these posts were retweeted less or more than expected. We employ 10 machine learning $(\mathrm{ML})$ algorithms to estimate the expected number of retweets based on 8 features of each tweet from historical data since President Trump was elected: number of likes, word count, readability, polarity, subjectivity, presence of link or multimedia content, time of day of posting, and number of days since Trump's election. Our results indicate agreement from all $10 \mathrm{ML}$ algorithms that the three flagged tweets for which we had retweet data were retweeted at higher rates than expected. These results suggest that flagging tweets by government officials might be counterproductive towards the spread of content deemed objectionable by social media platforms.
\end{abstract}




\section{Introduction}

\section{Background}

Spirited debate continues on how social media companies should handle cases of misinformation, incitement of violence, or otherwise objectionable content on their platforms (Dickerson, 2009), particularly when such posts emanate from representatives of national governments or other public officials (Yue, 2019). The debate pits roughly two sides against each other (Land, 2019). For purposes of simplification, one side might be said to believe that objectionable posts should not be deleted because they retain news value in that they represent influential views. Further, particularly in the United States, a view persists that social media platforms taking any action on posts deemed objectionable represents censorship and might contravene the spirit or even the text of the first amendment of the constitution that guarantees freedom of expression (Dickerson, 2009). The other side of this debate can be said to advocate that inaccurate and/or inflammatory posts must be deleted or flagged to prevent the spread of misinformation, or to prevent the incitement of violence (Pennycook \& Rand, 2019). After all, misinformation imposes high costs on society, from increases in healthcare costs and deaths in the case of antivaccine misinformation to destabilization of society from climate change related misinformation (Lewandowsky et al., 2012). Thus, strong tension exists in terms of balancing newsworthiness of content posted by public officials and potential damage emanating from such content.

Twitter and Facebook, as two of the most influential social networks in the US (Shearer, 2018), feature prominently in this debate and for a long time have not taken action on posts by US public officials. That situation largely changed in May 2020, when Twitter flagged a thread of two tweets by President of the US Donald Trump (hereinafter POTUS). In the tweet, POTUS claimed that use of mail-in ballots would lead to a rigged (2020 US presidential) election, ostensibly to favor his political opponents, and he asserted his opposition to their use. Twitter flagged that thread with a link titled "Get the facts about mail-in ballots" which pointed to a site with more in-depth information about mail-in ballots. Since then, Twitter has flagged three more tweets from POTUS, two of which it stated violated its policies on incitement to violence, and one of which was initially 
flagged as manipulated media but was eventually deleted after a copyright violation notice was filed against it.

This paper examines whether preliminary evidence supports that flagging tweets by POTUS reduces their spread as measured by the number of retweets. It is instructive to examine Twitter's intent in taking these actions on the tweets they found objectionable. CEO Jack Dorsey intimated that Twitter's intention in flagging the tweets was not to be "the arbiter of truth" but "to connect the dots of conflicting statements and show the information in dispute so people can judge for themselves" (Jack on Twitter, 2020). Twitter further added its belief that "those Tweets could confuse voters about what they need to do to receive a ballot and participate in the election process" (Twitter Safety on Twitter, 2020). In the case of the third tweet, Twitter explained that the "Tweet violated the Twitter Rules about glorifying violence. However, Twitter has determined that it may be in the public's interest for the Tweet to remain accessible." Table 1 summarizes the contents of the 5 flagged tweets and the justifications issued by Twitter for flagging each tweet.

Table 1: Flagged POTUS Tweets and Justifications for Flagging

\begin{tabular}{|l|l|l|}
\hline Tweet label & Tweet content (Link to tweet) & $\begin{array}{l}\text { Justification by Twitter for flagging } \\
\text { the tweet (Link to justification) }\end{array}$ \\
\hline A & $\begin{array}{l}\text { There is NO WAY (ZERO!) that } \\
\text { Mail-In Ballots will be anything less } \\
\text { than substantially fraudulent. } \\
\text { Mailboxes will be robbed; ballots will } \\
\text { be forged \& even illegally printed } \\
\text { out \& fraudulently signed. The } \\
\text { Governor of California is sending } \\
\text { Ballots to millions of people, } \\
\text { anyone.... } \\
\text { (https://twitter.com/realDonaldTrum } \\
\text { p/status/1265255835124539392) }\end{array}$ & $\begin{array}{l}\text { We added a label to two } \\
\text { California's vote-by-mail plans as part of } \\
\text { policy. We believe those Tweets could } \\
\text { confuse voters about what they need to } \\
\text { do to receive a ballot and participate in } \\
\text { the election process. }\end{array}$ \\
& $\begin{array}{l}\text { We also wanted to provide additional } \\
\text { context and conversation with regard to } \\
\text { voter fraud and mail-in ballots. We have } \\
\text { they are or how they got there, will } \\
\text { get one. That will be followed up } \\
\text { with professionals telling all of these } \\
\text { people, many of whom have never } \\
\text { even thought of voting before, how, } \\
\text { and for whom, to vote. This will be a } \\
\text { cases we add labels that link to more } \\
\text { Rigged Election. No way! } \\
\text { (https://twitter.com/realDonaldTrum } \\
\text { p/status/1265255845358645254) }\end{array}$ & $\begin{array}{l}\text { (https://twitter.com/i/events/1265330601 } \\
\text { 034256384) }\end{array}$ \\
\hline
\end{tabular}




\begin{tabular}{|c|c|c|}
\hline C & $\begin{array}{l}\text { https://twitter.com/i/status/12737706 } \\
69214490626 \\
\text { (https://twitter.com/realDonaldTrum } \\
\text { p/status/1273770669214490626) }\end{array}$ & $\begin{array}{l}\text { Manipulated media } \\
\text { - In September } 2019, \text { CNN reported on } \\
\text { a viral video about a friendship between } \\
\text { two toddlers } \\
\text { - On Thursday, the president shared a } \\
\text { version of the video which many } \\
\text { journalists confirmed was edited and } \\
\text { doctored with a fake CNN chyron } \\
\text { (https://twitter.com/i/events/1273790055 } \\
513903104 \text { ) }\end{array}$ \\
\hline D & $\begin{array}{l}\text {... These THUGS are dishonoring } \\
\text { the memory of George Floyd, and I } \\
\text { won't let that happen. Just spoke to } \\
\text { Governor Tim Walz and told him } \\
\text { that the Military is with him all the } \\
\text { way. Any difficulty and we will } \\
\text { assume control but, when the } \\
\text { looting starts, the shooting starts. } \\
\text { Thank you! } \\
\text { (https://twitter.com/realDonaldTrum } \\
\text { p/status/1266231100780744704) }\end{array}$ & $\begin{array}{l}\text { This Tweet violated the Twitter Rules } \\
\text { about glorifying violence. However, } \\
\text { Twitter has determined that it may be in } \\
\text { the public's interest for the Tweet to } \\
\text { remain accessible. } \\
\text { (https://twitter.com/realDonaldTrump/sta } \\
\text { tus/1266231100780744704) }\end{array}$ \\
\hline$E$ & $\begin{array}{l}\text { There will never be an "Autonomous } \\
\text { Zone" in Washington, D.C., as long } \\
\text { as I'm your President. If they try, } \\
\text { they will be met with serious force! } \\
\text { (https://twitter.com/realDonaldTrum } \\
\text { p/status/1275409656488382465) }\end{array}$ & $\begin{array}{l}\text { This Tweet violated the Twitter Rules } \\
\text { about abusive behavior. However, } \\
\text { Twitter has determined that it may be in } \\
\text { the public's interest for the Tweet to } \\
\text { remain accessible. } \\
\text { (https://twitter.com/realDonaldTrump/sta } \\
\text { tus/1275409656488382465) }\end{array}$ \\
\hline
\end{tabular}

Table 1 shows that reasons for flagging tweets are varied, from curtailing the spread of misinformation to preventing incitement of violence. Given that the justification for flagging the tweets points to what Twitter views as objectionable content for varied reasons: false information, manipulated content, and possible incitement of violence, a natural question concerns whether any effect exists on the degree to which the posted tweets are then shared with other people. An important consideration is the Streisand effect, whereupon the publicity of flagging content generates increased interest in the content, which might run counter to the goals of flagging the tweet in the first place (Jansen \& Martin, 2015). In this paper we examine whether the flagged tweets were shared at reduced rates than expected. 
We use machine learning models to achieve our research objective. We surmise that the number of retweets on a particular tweet by POTUS can be estimated by training various machine learning regression algorithms on historical data. Since he was elected, POTUS has tweeted more than 17,000 times, which is sufficient data to derive mathematical relationships among various variables that influence the number of retweets. After deriving these relationships using 10 machine learning algorithms, we score each of three tweets $-A, B$, and $C$ ( $D$ and $E$ were hidden by Twitter) - using the algorithms to estimate what would have been the number of retweets without the flagging. Our results suggest that the flagged tweets were retweeted at greater rates than expected, supporting the hypothesis that when Twitter flags tweets by POTUS, they will probably be retweeted more than they would have. Each of the 10 algorithms that we used predicted fewer than the observed number of retweets on the three flagged tweets for which we had retweet data. Given that Twitter's justification for flagging tweets is that the tweets are objectionable in the first place, if the tweets are then shared much more than would have been, it is important to ask whether flagging tweets is an effective mechanism for limiting the spread of content deemed objectionable by the content provider.

\section{Why Machine Learning}

This paper delves into the effect of flagging tweets by an influential individual, perhaps the most influential person in the world. To date, as noted earlier, POTUS has tweeted more than 17,000 times since his election, and of these only five have been flagged for reasons of misinformation, manipulated content, and "glorification of violence". Of the five, two were hidden by default from the public although the tweets were still accessible. These hidden tweets did not display the necessary information that we used for estimating the number of expected retweets using machine learning; thus, we were left with three tweets to score. Traditional methods of statistical analysis (e.g., ANOVA and MANOVA) would not be helpful in quantifying the effect of this flagging given how unlikely it is that Twitter would ever flag a huge mass of POTUS's tweets that would rectify the imbalance between flagged and unflagged tweets. Using machine learning, however, we can estimate the number of retweets on each of the flagged tweets based on the tweet's features and historical data. 
It is important to note what machine learning allows us to and to not do. In deriving the relationship between various features of a tweet and the number of times it is retweeted, we are creating a model of how Twitter users share POTUS tweets. Although the results of linear regression give some indication as to how the various features influence retweets, this is not the primary goal of our study. The linear regression results do provide important information regarding how Twitter users retweet POTUS's tweets, but at this point we do not aim to generalize these relationships to the rest of Twitter users' retweets. Another important thing to note is that this study is exploratory and presents the preliminary evidence regarding how the three of five flagged POTUS tweets have been retweeted. If it turns out that over time, Twitter or Facebook would flag a significant proportion of POTUS's or other government officials' tweets, it would be possible to conduct systematic analyses of the effect of flagging these tweets. For now, we can only estimate the effect of flagging of these few tweets on the number of retweets. This should not subtract from the value of the study. POTUS's tweets are consequential no matter the number that gets flagged. Given that POTUS is arguably the most influential individual in the world, it is important to investigate any indication that flagging tweets deemed inaccurate, inflammatory, or objectionable may be counterproductive if it leads to higher levels of sharing than expected.

In the next section we outline some related research and theory to this study. We proceed to describe our methods of data collection and analyses before discussing results, implications of the study for theory and practice, and concluding.

\section{Related Research and Theory}

In this section, we first explain how information processing in social media is different than in other contexts. We describe some salient issues and problems, such as fake news, misinformation, and online violence and hatred, resulting from the use of and information processing in social media. We then discuss theories of confirmation bias and cognitive dissonance and how they have been utilized by social media platforms to employ design changes such as content flagging and source rating to tackle the problems. We then focus on content flagging and discuss and explain its potential impact on the issues. Finally, we 
identify the gaps and explain how the present study differs from and contributes to the existing literature.

\section{Information Processing in Social Media and Attendant Social Media Issues}

Information processing and consuming news on social media are different than other contexts in several ways. First and foremost, the mindset of the users is different. On social media, most users read information and news for fun and entertainment (Kim and Dennis, 2019). In other words, they possess a hedonic mindset and their primary goal using social media is for pleasure and enjoyment. In contrast, users of other Internet contexts often possess a utilitarian mindset (Moravec et al., 2019). Users of e-commerce sites (e.g., Amazon), for example, read product and website information (e.g., product review) in order to make a purchase decision; and users of many online communities (e.g., Quora) consume information to get answers to their questions. Second, compared to the traditional news media, the source of information on social media is often unclear. This is because social media sites such as Facebook and Twitter use various machine learning algorithms to push information and news to the users, combined with advertisements and trends. These algorithm-decided information and news are very intermixed, some from reputable sources and some from disreputable sources (Moravec et al., 2019). Third, users on social media are often presented with massive information and news that are constantly updated. As a result, they are less likely to devote significant time and effort to understand and reflect on each piece of information they have read.

Because of these context-specific factors, several issues and problems have been prevailing on social media including fake news and misinformation as well as online violence and hatred (Kim et al., 2019; Eagle et al., 2002; Grosseck et al., 2012). Fake news and misinformation particularly manifest on social media given the large volume of information from sources that are difficult for users to identify. Moreover, because of the hedonic mindset of the users, they are reluctant to invest the effort to research the sources of many fake and misleading news stories that are carefully crafted (Kim and Dennis, 2019; Moravec et al., 2019). Consequently, fake news and misinformation on social media have been misleading people, maligning people and communities, and/or spreading false propaganda (Panigrahi and Singh, 2017). Apart from the fake news and 
misinformation, the social media context enables violence and hatred to flourish. Crime networks and extremist groups worldwide have been increasingly inciting violence and preaching hate on social media sites given their ubiquity and low cost (Swibel and Boms, 2018). Some white nationalists even openly operate on social media platforms to instigate and fuel violence and communal hatred. Richard Spencer who has over 75,000 followers on Twitter, for instance, organized the "Unite the Right" far-right Charlottesville Rally (Siegel, 2019).

\section{Creating Cognitive Dissonance and the Effects of Content Flagging}

Increasing concerns over these issues and problems have led both academia and industry to study and develop various approaches to combat them (e.g., Kim and Dennis, 2019; Colliander, 2019; Jang et al., 2018; Jang and Kim, 2018; Kim et al., 2019). These studies are primarily based on two general theories of confirmation bias and cognitive dissonance. Confirmation bias generally refers to people preferring information that matches their prior beliefs (Devine et al., 1990; Minas et al., 2014; Nickerson, 1998). This is because rejecting the new information that challenges one's preexisting beliefs is simpler than reassessing them (Kim and Dennis, 2019). Notably, confirmation bias is exacerbated when individuals are in a hedonic mindset (Hirschman and Holbrook, 1982). As a result, misinformation, hate speech, and violent content on social media can be widely endorsed and spread among users who possess the same existing beliefs. One means of interrupting confirmation bias is to create cognitive dissonance (Festinger, 1962; Mills, 1999). Cognitive dissonance occurs when individuals encounter two pieces of contradictory information that cannot be readily reconciled (Moravec et al., 2019). Based on this, two major approaches have been developed and employed to combat the foregoing issues and problems including content flagging and source reputation ratings. Source reputation ratings, for example, have been found to have a direct influence on social media users' believability by mitigating the confirmation bias (Kim et al., 2019). Likely, adding a flag saying a news story is false will create cognitive dissonance when users want to believe the news story that matches their prior beliefs (Moravec et al., 2019). In the present study, we focus on the approach of flagging and examine its influence on social media users' response to flagged content. 
As noted earlier, the research question we seek to answer by focusing on content flagging on social media is: does flagging POTUS's tweets lead to fewer or more retweets? Or in other words, does flagging mitigate or worsen the foregoing issues regarding POTUS's tweets? On one hand, it is rational for one to expect that flagging should mitigate the problem because of the cognitive dissonance concept discussed above. Flagging a tweet helps create a cognitive dissonance that forces some users to resolve the inconsistency (between their beliefs and the contradictory facts implied by the flag) before responding to the tweet (e.g., retweeting, liking, and commenting). Consequently, some users may choose to not retweet as they perceive the flag is more plausible. Also, some extant research suggests that social media users will delete posts that have been flagged as containing erroneous information. Shared content containing misinformation is likely to be deleted after it is labeled as such, because posters may not want to spread or to be associated with spreading misinformation (Friggeri et al., 2014). Overall, these considerations imply that flagging tweets would reduce the amount of times the tweet would be shared.

On the other hand, there are several factors that suggest otherwise. First, the Streisand effect ${ }^{1}$ suggests there may be increased attention to a tweet as a result of attempting to suppress the tweet. This can be especially true considering that, to date, only a few of POTUS's tweets have been flagged, making these flagged tweets outliers for the users. Therefore, when Twitter flags the content of a tweet for whichever reason, the flagging in itself is likely a newsworthy event. This means that flagging content might prove counterproductive for the purposes of limiting the spread of objectionable content. Although it may not be explicitly stated by Twitter that it seeks to reduce the spread of that content, it is reasonable to infer that Twitter would not seek to encourage the spread of information that it deems inaccurate, inflammatory and/or otherwise objectionable. Second, in the case of the highly polarized political atmosphere like the US (lyengar et al., 2019), it is not immediately clear that a social media platform flagging POTUS's posts should lead to the posts being shared less. While generally flagging posts should reduce their resharing, the publicity of flagging POTUS's posts might attract even more attention to the post and encourage his political supporters to share them as an act of solidarity. Taken together, these conflicting considerations warrant our study. 
To summarize, our study differs from extant literature in several ways. First, existing studies are primarily focused on the flagging of fake news (e.g., Colliander, 2019; Kim et al., 2019; Moravec et al., 2019). While they are, to some extent, related to each other, fake news is different than content that glorifies violence and preaches hate in that the former has often been instrumental in instigating the latter (Panigrahi and Singh, 2017). By focusing on both potential misinformation and incitement to violence, we contribute to conferring a holistic picture of how content flagging would impact the combating of misinformation and violent content on social media. Second, prior studies have largely focused on the influence of content flagging on social media users' beliefs (e.g., Kim and Dennis, 2019; Kim et al., 2019; Moravec et al., 2019). This study, in contrast, examines how users would actually act differently facing flagged content as opposed to the content that is not flagged. Third, unlike these studies that have relied on experimental design and studies to investigate the effect of content flagging, we employ a machine learning method that aligns more with the current approaches and mechanisms used by the industry. Finally, as discussed earlier, we only focus on the flagging of content created by POTUS. Given the extraordinary influence and status of this particular person, our study may reveal some differentiated and nuanced outcomes that have been neglected by the prior research. Our study would shed light on whether flagging content may inadvertently generate greater publicity for the content than would otherwise be expected.

\section{Method}

To accomplish our research objective for this paper, we first learn from the data the relationship between the likes on POTUS's tweets and their retweets, while controlling for several variables. We employ 10 different machine learning regression algorithms to derive this relationship. We proceed to compare the algorithms' performances based on how their predictions deviate from the observed retweets. Next, we score each of the three flagged tweets against the generated models to derive estimates of retweet counts. Last, we compare the observed retweet counts with the predicted retweets from the

models. The difference is our estimate of the effect of flagging POTUS's tweets on retweeting. 


\section{Data Collection}

We collected US President Trump's tweets from since he was elected on 8 November 2016, until 8 June 2020. Data collection took place on 8 June 2020. We only collected those tweets that Trump himself posted and excluded his retweets of others' tweets. Overall, there were 17,212 such tweets during the relevant period. The data included the tweet ID, the text content of the tweet, date and time of posting, the number of likes on the tweet, and the number of retweets. We collected this data from Trump Twitter Archive (Trump Twitter Archive, 2020), a site which collects and updates data about tweets posted from the @realDonaldTrump account in real time. This archive has been used in academic research before to derive POTUS's linguistic style when using Twitter (Clarke \& Grieve, 2019).

\section{Data Analysis}

We surmise that the number of POTUS's retweets on a given tweet may be estimated as a function of the number of likes on the tweet, its readability, its word count, its polarity, and the time of day of posting the tweet. We built our models using a variety of regression algorithms, 10 in total: neural network, support vector machine, k nearest neighbors, linear, ridge, lasso, decision tree, gradient boosting tree, aggregate bagging tree, and random forest. The data set was split $70 \%$ and $30 \%$ training set and testing set respectively for all algorithms. We employed feature scaling by transforming each value to the corresponding z-score for the column. Table 2 below summarizes the algorithms and parameters used to examine our hypotheses. All analyses were done using Python's Scikit-learn package (Pedregosa et al., 2011).

Table 2: Study's Training Algorithms and Parameters

\begin{tabular}{|l|l|l|}
\hline Algorithm & Description & Key Parameters \\
\hline $\begin{array}{l}\text { Neural } \\
\text { network }\end{array}$ & $\begin{array}{l}\text { Algorithm modeled on how the human } \\
\text { brain works, with higher level features } \\
\text { from shallow layers gradually } \\
\text { decomposed to lower level features in } \\
\text { the deeper layers (Glorot \& Bengio, } \\
\text { 2010). }\end{array}$ & $\begin{array}{l}\text { Multiple layer perceptron feedforward } \\
\text { network, 2 hidden layers of 7 nodes } \\
\text { each, } \\
\text { ReLU activation function, } \\
\text { L2 regularization penalty 0.0001, } \\
\text { and mean squared error loss function. }\end{array}$ \\
\hline Support & Algorithm that solves $w$ (parameter & Linear kernel \\
\hline
\end{tabular}




\begin{tabular}{|c|c|c|}
\hline $\begin{array}{l}\text { vector } \\
\text { machine }\end{array}$ & $\begin{array}{l}\text { vector) and } b \text { (bias) while minimizing } \\
\text { regression risk (Yang et al., 2002) }\end{array}$ & \\
\hline $\begin{array}{l}\text { K nearest } \\
\text { neighbors }\end{array}$ & $\begin{array}{l}\text { Memory-based algorithm that } \\
\text { estimates the target value of an } \\
\text { observation based on a similarity } \\
\text { measure, i.e., a pre-specified K or } \\
\text { number of observations closest to the } \\
\text { given observation (Cover \& Hart, } \\
\text { 1967). }\end{array}$ & $K=17$ \\
\hline Linear & $\begin{array}{l}\text { Algorithm that finds a linear } \\
\text { relationship between input features } \\
\text { and target feature. It finds input } \\
\text { parameters which minimize the } \\
\text { squares of the deviation of predicted } \\
\text { values from observed values(Forkuor } \\
\text { et al., 2017). }\end{array}$ & Ordinary least squares regression \\
\hline Ridge & $\begin{array}{l}\text { An extension of linear regression } \\
\text { except there is a regularization } \\
\text { parameter - the L2 penalty - to reduce } \\
\text { the gradients of the relationships } \\
\text { between input features and the target } \\
\text { features. It minimizes the sum of } \\
\text { squares of the deviation of predicted } \\
\text { values from observed values plus the } \\
\text { product of the regularization } \\
\text { parameter and the square of each } \\
\text { parameter (Marquardt \& Snee, 1975). }\end{array}$ & Alpha $=100$ \\
\hline Lasso & $\begin{array}{l}\text { Similar to ridge but utilizes the L1 } \\
\text { penalty to reduce the size of each } \\
\text { input feature parameter. It minimizes } \\
\text { the sum of squares of the deviations of } \\
\text { predicted values from observed values } \\
\text { plus the product of the regularization } \\
\text { parameter and the absolute value of } \\
\text { each parameter (Tibshirani, 1996). }\end{array}$ & Alpha $=0.1$ \\
\hline $\begin{array}{l}\text { Decision } \\
\text { tree }\end{array}$ & $\begin{array}{l}\text { A greedy algorithm that repeatedly } \\
\text { splits the dataset until the highest } \\
\text { purity levels are achieved for each } \\
\text { given leaf; for regression problems, a } \\
\text { given node is purer than another if it } \\
\text { has a lower mean square or absolute } \\
\text { error with respect to the target variable } \\
\text { (Loh, 2011). }\end{array}$ & $\begin{array}{l}\text { Maximum depth }=4 \text {, splitting criterion } \\
=\text { mean square error. }\end{array}$ \\
\hline $\begin{array}{l}\text { Gradient } \\
\text { boosting }\end{array}$ & $\begin{array}{l}\text { Ensemble algorithm using a series of } \\
\text { weak learners to build the regression }\end{array}$ & $\begin{array}{l}\text { Maximum depth }=4 \text {, learning rate }= \\
0.1, \text { loss function }=\text { least squares }\end{array}$ \\
\hline
\end{tabular}




\begin{tabular}{|l|l|l|}
\hline tree & $\begin{array}{l}\text { tree, with emphases on erroneously } \\
\text { predicted observations at each } \\
\text { iteration (Natekin \& Knoll, 2013). }\end{array}$ & \\
\hline $\begin{array}{l}\text { Bootstrap } \\
\text { aggregating } \\
\text { (bagging) } \\
\text { tree }\end{array}$ & $\begin{array}{l}\text { Training sets are created using } \\
\text { bootstrap - sampling with replacement } \\
\text { - and trees are grown without pruning } \\
\text { (Prasad et al., 2006). }\end{array}$ & $\begin{array}{l}\text { Maximum depth = 4, number of } \\
\text { candidate stumps = 10, maximum } \\
\text { number of features for splitting }=1\end{array}$ \\
\hline $\begin{array}{l}\text { Random } \\
\text { forest }\end{array}$ & $\begin{array}{l}\text { Similar to bagging in that it uses } \\
\text { bootstrap sampling of the data at each } \\
\text { iteration, but uses a subset of features } \\
\text { to recursively split the nodes. After N } \\
\text { iterations, there are N candidate trees } \\
\text { that constitute the forest. Validation of } \\
\text { the data is done using the out-of-bag } \\
\text { sample (Prasad et al., 2006) }\end{array}$ & $\begin{array}{l}\text { Maximum depth }=4, \text { number of } \\
\text { candidate trees }=100, \text { splitting } \\
\text { criterion = mean squared error, } \\
\text { maximum number of features for } \\
\text { splitting = 8 }\end{array}$ \\
\hline
\end{tabular}

\section{Feature Descriptions}

We employed feature engineering to generate new features based on the raw features provided from the data. From the content of each tweet, we derived the word count, readability, polarity, and subjectivity of the text as potential features influencing the number of retweets. We removed URLs from the tweets to generate accurate values for word count, readability, polarity, and subjectivity of each tweet. Further, we also surmised that content with images or videos or links to external sources of information might be retweeted differently than purely text content; thus, we engineered a new feature labeled "Link_Present" the value of which indicated whether the link contained a link or image or video. Another feature we engineered for each tweet was the number of days since POTUS was elected, on the rationale that over time POTUS probably acquired new followers leading to more retweets of his tweets; this suggests that over time POTUS's tweets are more likely to be retweeted (Clarke \& Grieve, 2019). We describe each of the features in the following subsections.

Retweets. A retweet is a mechanism by which users share existing tweets to their own followers, and it is primarily how information spreads on Twitter (Kupavskii et al., 2012). The number of retweets on a given tweet forms the dependent variable for this study. 
Likes. On Twitter, users may click the heart-shaped like icon to indicate positive feelings about a given tweet (Valenzuela et al., 2018). The number of likes on a given tweet is an independent variable in our model.

Readability. The readability of a piece of text is the level of ease or difficulty with which text material can be understood by a particular reader who is reading that text for a specific purpose (Pikulski, 2002). We measure readability using the Flesch-Kincaid readability scale with higher measures indicating greater readability (Flesch, 1948).

Word Count. The number of words in a given tweet might also influence how it is shared; thus, we control for this variable in our model.

Polarity. The polarity of a piece of text measures the extent to which the overall text's sentiment is negative or positive (Wilson et al., 2005). Using the TextBlob package in Python (Loria, 2020), the polarity of a text varies between -1 and 1 ; negative measures indicate negative sentiment, 0 indicates neutral sentiment, and positive measures indicate positive sentiment.

Subjectivity. The subjectivity of a piece of text is the extent to which it expresses an opinion (Liu, 2010). Subjectivity varies from 0 (neutral opinion) to 1 (strongest opinion) using the TextBlob package.

Hour of Day. We surmised that the time of day when a tweet is posted might also have a bearing on its popularity. For our purposes, we measured the time as the hour on Eastern Standard Time, given that it's the time zone of Washington DC where the president resides.

Table 3 describes the variables and their descriptive statistics.

Table 3: Descriptive Statistics

\begin{tabular}{|l|l|l|l|l|l|}
\hline Variable & Description & Mean & $\begin{array}{l}\text { Standard } \\
\text { Deviation }\end{array}$ & Minimum & Maximum \\
\hline Retweets & $\begin{array}{l}\text { Number of times the } \\
\text { tweet was shared. }\end{array}$ & $20,963.97$ & $13,764.61$ & 1 & $369,530.00$ \\
\hline Likes & $\begin{array}{l}\text { Number of times the } \\
\text { tweet was liked or } \\
\text { favorited. }\end{array}$ & $89,922.47$ & $55,573.57$ & 1 & $879,647.00$ \\
\hline Word_Count & $\begin{array}{l}\text { Number of words in a } \\
\text { tweet. }\end{array}$ & 27.93 & 16.31 & 1 & 60 \\
\hline
\end{tabular}




\begin{tabular}{|l|l|l|l|l|l|}
\hline Readability & $\begin{array}{l}\text { Level of ease or difficulty } \\
\text { with which the tweet can } \\
\text { be read. Measured using } \\
\text { the Flesch-Kincaid scale. }\end{array}$ & 49.46 & 56.47 & -514.3 & 206.84 \\
\hline Time_of_Day & $\begin{array}{l}\text { Hour of day (US Eastern } \\
\text { Standard Time) when } \\
\text { tweet was posted. }\end{array}$ & 12.93 & 5.63 & 0 & 23 \\
\hline Polarity & $\begin{array}{l}\text { The extent to which the } \\
\text { overall text's sentiment is } \\
\text { negative or positive. }\end{array}$ & 0.15 & 0.34 & -1 & 1 \\
\hline Subjectivity & $\begin{array}{l}\text { The extent to which the } \\
\text { overall text's sentiment is } \\
\text { neutral or opinionated. }\end{array}$ & 0.46 & 0.28 & 0 & 1 \\
\hline $\begin{array}{l}\text { Days_Since_- } \\
\text { Elected }\end{array}$ & $\begin{array}{l}\text { Number of days since } \\
\text { POTUS was elected at } \\
\text { the time the tweet was } \\
\text { posted }\end{array}$ & 512.23 & 359.57 & 0 & $1,308.00$ \\
\hline Link_Present & $\begin{array}{l}\text { Whether a link or image } \\
\text { or video was present in } \\
\text { the tweet }\end{array}$ & 0.32 & 0.46 & 0 & 1 \\
\hline
\end{tabular}

We checked for collinearity using the variance inflation factor (VIF). This factor ranged from 1.05 to 1.73 for each independent variable, falling significantly below the threshold of 10 that indicates multicollinearity to be a problem (Chatterjee \& Hadi, 2015). Our data collection and analyses process are summarized in Figure 1.

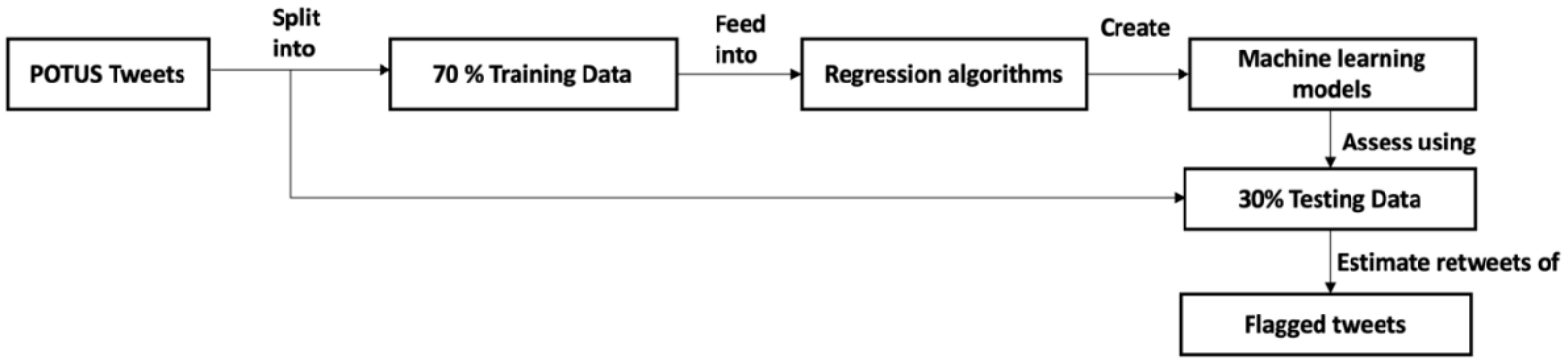

Figure 1: Study's Data Collection and Analyses Process

\section{Results}

In Table 4 we show the root mean square error (RMSE), mean absolute error, and rsquared value (coefficient of determination) for the testing sets for each of the algorithms. The RMSE is the square root of the average of the squares of the deviations of all the observations from each algorithm's prediction of the relevant tweet's retweets. The mean 
absolute error (MAE) is the average of all the absolute deviations of the predicted retweets from the observed retweets. An algorithm with a lower RMSE performs better than that with a higher RMSE, and this is the same for the MAE, because in general these show that the predicted retweets do not deviate as much from the observed retweets. The $r-$ squared value quantifies the amount of variation in the retweets that can be explained by the variation in the predictors; hence, the higher the r-squared value the better.

The results show that gradient boosting, neural network, and bagging performed better than the other algorithms based on the RMSE (0.31 - 0.33), MAE (0.18), and rsquared values $(0.86-0.88)$, and that these five performed basically identically. Further, these three algorithms predicted that the first flagged tweet (Tweet $A$ ) would have been retweeted between 32,029 (neural network) and 34,846 (gradient boosting) times; the observed number of retweets was 38,549. For Tweet B, which was retweeted 21,545 times, the three algorithms predicted between 16,135 (neural network) and 20,699 (bagging) retweets. And last, whereas Tweet $C$ was retweeted 215,600 times, the three algorithms predicted between 69,035 (bagging) and 199,257 (neural network) retweets. All told, the 10 algorithms agreed that the estimated number of retweets fell below the observed number of retweets for the flagged tweets. Tweets $A$ and $B$ were retweeted 1.2 and 1.12 times than the average prediction of all 10 algorithms respectively, and Tweet $C$ was retweeted 1.75 times the equivalent number.

Table 4: Ten ML Algorithms: Comparison of Performance Using RMSE, MAE, and RSquared, and Predictions of Retweets of Flagged POTUS Tweets

\begin{tabular}{|l|l|l|l|l|l|l|l|l|l|}
\hline Regression Algorithm & RMSE & MAE & $\mathbf{R}^{\mathbf{2}}$ & PR_A & ER_A & PR_B & ER_B & PR_C & ER_C \\
\hline Gradient Boosting & 0.31 & 0.18 & 0.88 & 34,846 & 3,703 & 19,461 & 2,084 & 197,545 & 18,055 \\
\hline Neural Network & 0.32 & 0.18 & 0.87 & 32,029 & 6,520 & 16,135 & 5,410 & 199,257 & 16,343 \\
\hline Bagging & 0.33 & 0.18 & 0.86 & 34,803 & 3,746 & 20,699 & 846 & 69,035 & 146,565 \\
\hline Support Vector Machine & 0.34 & 0.20 & 0.85 & 31,894 & 6,655 & 18,652 & 2,893 & 104,503 & 111,097 \\
\hline Ridge & 0.34 & 0.20 & 0.85 & 33,252 & 5,297 & 19,388 & 2,157 & 109,723 & 105,877 \\
\hline Linear & 0.34 & 0.20 & 0.85 & 33,437 & 5,112 & 19,436 & 2,109 & 110,915 & 104,685 \\
\hline Random Forest & 0.35 & 0.21 & 0.84 & 30,708 & 7,841 & 18,779 & 2,766 & 125,107 & 90,493 \\
\hline Lasso & 0.35 & 0.21 & 0.84 & 29,537 & 9,012 & 18,955 & 2,590 & 96,714 & 118,886 \\
\hline Decision Tree & 0.37 & 0.22 & 0.82 & 33,827 & 4,722 & 19,292 & 2,253 & 85,081 & 130,519 \\
\hline K Nearest Neighbors & 0.39 & 0.25 & 0.80 & 29,561 & 8,988 & 21,187 & 358 & 130,933 & 84,667 \\
\hline
\end{tabular}


RMSE: Root Mean Square Error; MAE: Mean Absolute Error; PR_A: Predicted_Retweets for Tweet A; ER_A: Excess Retweets for Tweet A; PR_B: Predicted_Retweets for Tweet B; ER_B: Excess Retweets for Tweet B; PR_C: Predicted_Retweets for Tweet C; ER_C: Excess Retweets for Tweet C.

\section{Discussion}

In this study, we set out to examine preliminary evidence as to the effect of the flagging of tweets by POTUS on retweets. Using 10 machine learning algorithms we estimated the number of retweets on POTUS's tweets based on 8 features: number of likes, word count, readability, polarity, subjectivity, presence of link or multimedia content, time of day of posting, and number of days since POTUS was elected. The 10 algorithms all had high R-squared values ranging from 0.80 to 0.88 . Although the algorithms' predictions of the number of retweets varied, all the estimates suggest that each of the three flagged tweets was retweeted at higher than expected values based on the input features. These are unexpected findings given that existing studies and theories (such as cognitive dissonance) largely suggest that flagged tweets (because of being misleading and/or inflammatory) would be less likely to be retweeted. Also, to the extent that content that is flagged as containing false information is likely to be deleted because many social media users do not want to propagate or be associated with spreading false information (Friggeri et al., 2014), our findings are new and unexpected. So, what could be the potential explanations for such findings?

There are at least three potential explanations. First, as discussed earlier, the Streisand effect would offer an explanation if the efforts to suppress information inadvertently bring more attention to it. Second, the status and extraordinary influence of POTUS may also play a factor here considering the polarized nature of US politics and POTUS's enthusiastic base of supporters (Smith \& Hanley, 2018). Hence when Twitter flags any of POTUS's tweets, the ensuing publicity may lead to the flagged tweets being reshared even more than expected. POTUS's political opponents may also share flagged tweets because the rationale for flagging the tweets paints him in negative light. It is also important to note that prominent journalists and news outlets may also share flagged tweets because of their news value and thus spread the flagged content to an even broader national and global audience. And instances of this phenomenon can be seen in news articles in the New York Times, BBC, Washington Post, and CNN which covered 
news of the flagged tweets and embedded the tweets in their articles (BBC, 2020; CNN, 2020; New York Times, 2020; Washington Post, 2020). Indeed, these news stories amount to free publicity and may further politically benefit POTUS even when they are deemed objectionable, as was the case in the 2016 presidential election (Ott \& Dickinson, 2019). Third, the findings may also be attributed to the unique setting and context of the present study. In prior studies such as Kim and Dennis (2019), Kim et al. (2019), and Moravec et al. (2019), for example, the authors have largely employed experiments with subjective primary data to examine the impact of content flagging in social media platforms. This study, by contrast, relies on the objective secondary data of Twitter, one of the most popular and influential social media platforms. Also, compared to these studies that tend to focus on the beliefs of the users and the believability of the content, our study focuses on the actual responses of users in a real context.

In light of our findings, should Twitter and other influential social media platforms continue to flag content that they deem objectionable? Or should they just rely on users to make their own judgments as to the veracity and appropriateness of posts by government officials? Twitter's justifications for flagging tweets $A$ and $B$ have been retweeted only 13,000 and 2,000 times respectively, compared to 50,000 and 23,000 for the original tweets. This implies that flagging content by POTUS, regardless of the justification, leads it to being re-shared more than the justification for flagging it in the first place. Our suggestion, based on our results, is that flagging content deemed objectionable may be counterproductive until it is done so often as to be un-newsworthy. Yet, considering that it is unlikely that social media platforms would ever flag content by the US president often enough that the flagging would not generate news value and draw attention to the flagged content, a more effective method might be to delete content that violates the platform's terms of service. The resistance to such drastic actions might be high, however (Dickerson, 2009; Ott \& Dickinson, 2019). Another alternative would be to keep flagging objectionable content while also restricting the ability to reshare that content to achieve the objective of limiting its spread. External stakeholders could also play a role here. Coca-Cola, one of the largest advertisers on social media platforms, for example, has announced that it will halt all the advertisements on Facebook, Instagram, and Twitter 
at least till December 31st, 2020, in order to pressure the social media companies to more stringently police disinformation and hate speech (Bhattacharjee and Graham, 2020).

\section{Conclusion}

To conclude, the present study contributes to the extant literature by exploring and presenting some preliminary evidence on how the flagged tweets of POTUS have been retweeted. Future research may confirm and/or complement our findings if over time, more and more tweets are flagged across the social media platforms and there are sufficient data for conducting a longitudinal and systematic analysis of social media content flagging. It is our hope that scholars will look beyond their own fields and employ various ideas, theories, and methods to study the various issues brought by social media.

\section{Footnotes}

${ }^{1}$ The Streisand effect is a name given to the phenomenon of increased attention to content as a result of attempting to suppress that content (Jansen and Martin, 2015). In 2003, for example, the singer and actress Barbara Streisand sued a photographer for invasion of privacy after he had posted a picture of her house to an online database of houses along the California coastline. The ensuing publicity led to the picture being downloaded 420,000 times in the next month, compared to just six times before the lawsuit. The lawsuit was dismissed by the courts (Jansen and Martin, 2015).

\section{References}

BBC. (2020). Twitter Hides Trump Tweet for "Glorifying Violence." BBC News (accessed May 29; https://www.bbc.com/news/technology-52846679

Bhattacharjee, R. \& Graham, M. (2020). Coca-Cola Pauses Advertising on all Social Media Platforms Globally, CNBC (accessed Jun 30, 2020; https://www.cnbc.com/2020/06/26/cocacola-pauses-advertising-on-all-social-media-platforms-globally.html

Chatterjee, S., \& Hadi, A. S. (2015). Regression Analysis by Example. John Wiley \& Sons.

Clarke, I., \& Grieve, J. (2019). Stylistic Variation on the Donald Trump Twitter Account: A Linguistic Analysis of Tweets Posted between 2009 and 2018. PLOS ONE, 14(9).

CNN. (2020). Twitter Puts Warning on Trump Tweet for "Threat of Harm" against DC Protesters. CNN (Accessed June 23; https://www.cnn.com/2020/06/23/tech/trump-twitter-violencewarning/index.html). 
Colliander, J. (2019). "This Is Fake News": Investigating the Role of Conformity to Other Users' Views When Commenting on and Spreading Disinformation in Social Media. Computers in Human Behavior, 97, 202-215.

Cover, T., \& Hart, P. (1967). Nearest Neighbor Pattern Classification. IEEE Transactions on Information Theory, 13(1), 21-27.

Devine, P. G., Hirt, E. R., \& Gehrke, E. M. (1990). Diagnostic and Confirmation Strategies in Trait Hypothesis Testing. Journal of Personality and Social Psychology, 58 (6), 952-963.

Dickerson, N. P. (2009). What Makes the Internet so Special-And Why, Where, How, and by Whom Should Its Content Be Regulated. The Thirteenth Annual Frankel Lecture-Comment. Houston Law Review, 46(1), 61-102.

Eagle, L., De Bruin, A. \& Bulmer, S. (2002). Violence, Values, and the Electronic Media Environment. Corporate Communications: An International Journal, 7(1), 25-33.

Forkuor, G., Hounkpatin, O. K. L., Welp, G., \& Thiel, M. (2017). High Resolution Mapping of Soil Properties Using Remote Sensing Variables in South-Western Burkina Faso: A Comparison of Machine Learning and Multiple Linear Regression Models. PLOS ONE, 12(1).

Friggeri, A., Adamic, L., Eckles, D., \& Cheng, J. (2014). Rumor Cascades. Eighth International AAAl Conference on Weblogs and Social Media.

Glorot, X., \& Bengio, Y. (2010). Understanding the Difficulty of Training Deep Feedforward Neural Networks. Proceedings of the Thirteenth International Conference on Artificial Intelligence and Statistics, 249-256.

Grosseck, G., Holotescu, C. \& Danciu, E. L. (2012). Violence 2.0: A Review of Social MediaBased Violence Experiences Among Teens. 3rd International Conference Social Work Perspective of Quasi-Coercive Treatment of Offenders "VIOLENCE AMONG ADOLESCENTS", UVT Timisoara, May 1-12.

Hirschman, E. C., \& Holbrook, M. B. (1982). Hedonic Consumption: Emerging Concepts, Methods and Propositions. Journal of Marketing, 46 (3), 92-101.

lyengar, S., Lelkes, Y., Levendusky, M., Malhotra, N., \& Westwood, S. J. (2019). The origins and consequences of affective polarization in the United States. Annual Review of Political Science, 22, 129-146.

Jack (2020). "This does not make us an 'arbiter of truth.' Our intention is to connect the dots of conflicting statements and show the information in dispute so people can judge for themselves. More transparency from us is critical so folks can clearly see the why behind our actions." Twitter (May 27; https://twitter.com/jack/status/1265837139360485376).

Jansen, S. C., \& Martin, B. (2015). The Streisand Effect and Censorship Backfire. International Journal of Communication, Vol,9, 656-671.

Kim, A. \& Dennis, A. R. (2019). Says Who? The Effects of Presentation Format and Source Rating on Fake News in Social Media. MIS Quarterly, 43(3), 1025-1039.

Kim, A., Moravec, P. L. \& Dennis, A.R. (2019). Combating Fake News on Social Media with Source Ratings: The Effects of User and Expert Reputation Ratings. Journal of Management Information Systems, 36(3), 931-968.

Kupavskii, A., Ostroumova, L., Umnov, A., Usachev, S., Serdyukov, P., Gusev, G., \& Kustarev, A. (2012). Prediction of Retweet Cascade Size Over Time. Proceedings of the 21st ACM International Conference on Information and Knowledge Management - CIKM '12, 2335. 
Land, M. K. (2019). 11 Regulating Private Harms Online: Content Regulation under Human Rights Law. Human Rights in the Age of Platforms, 285.

Lewandowsky, S., Ecker, U. K. H., Seifert, C. M., Schwarz, N., \& Cook, J. (2012). Misinformation and Its Correction: Continued Influence and Successful Debiasing. Psychological Science in the Public Interest, 13(3), 106-131.

Liu, B. (2010). Sentiment Analysis and Subjectivity. Handbook of Natural Language Processing, Vol.2, 627-666.

Loh, W. Y. (2011). Classification and Regression Trees. Wiley Interdisciplinary Reviews: Data Mining and Knowledge Discovery, 1(1), 14-23.

Loria, S. (2020). Textblob Documentation: Simplified Text Processing. TextBlob (Accessed July 4; https://textblob.readthedocs.io/en/dev/)

Marquardt, D. W., \& Snee, R. D. (1975). Ridge Regression in Practice. The American Statistician, 29(1), 3-20.

Mills, J. (1999). Improving the 1957 Version of Dissonance Theory, in Cognitive Dissonance: Progress on a Pivotal Theory in Social Psychology, E. Harmon-Jones and J. Mills (eds.), Washington, DC: American Psychological Association, 25-42.

Minas, R. K., Potter, R. F., Dennis, A. R., Bartelt, V., \& Bae, S. (2014). Putting on the Thinking Cap: Using Neurois to Under- stand Information Processing Biases in Virtual Teams. Journal of Management Information Systems, 30(4), 49-82.

Mo Jang, S., Geng, T., Li, J. Y. Q., Xia, R., Huang, C. T., Kim, H. \& Tang, J. (2018). A Computational Approach for Examining the Roots and Spreading Patterns of Fake News: Evolution Tree Analysis. Computers in Human Behavior, 84, 103-113.

Mo Jang, S. \& Kim, J. K. (2018). Third Person Effects of Fake News: Fake News Regulation and Media Literacy Interventions. Computers in Human Behavior, 80, 295-302.

Moravec, P. L., Minas, R.K. \& Dennis, A.R. (2019). Fake News on Social Media: People Believe What They Want to Believe When It Makes No Sense at All. MIS Quarterly, 43(4), 13431360.

Natekin, A., \& Knoll, A. (2013). Gradient Boosting Machines, A Tutorial. Frontiers in Neurorobotics, 7.

New York Times. (2020). Twitter Places Warning on Trump Minneapolis Tweet, Saying It Glorified Violence. (accessed July 4; https://www.nytimes.com/2020/05/29/technology/trump-twitterminneapolis-george-floyd.html)

Nickerson, R. S. (1998). Confirmation Bias: A Ubiquitous Phenomenon in Many Guises. Review of General Psychology, 2(2), 175-220.

Ott, B. L., \& Dickinson, G. (2019). The Twitter Presidency: Donald J. Trump and the Politics of White Rage. (1st ed.). Routledge.

Panigrahi, S. \& Singh, J. (2017). Deadly Combination of Fake News and Social Media. Rajiv Gandhi Institute for Contemporary Studies, New Delhi-110001.

Pedregosa, F., Varoquaux, G., Gramfort, A., Michel, V., Thirion, B., Grisel, O., Blondel, M., Prettenhofer, P., Weiss, R., \& Dubourg, V. (2011). Scikit-learn: Machine learning in Python. Journal of Machine Learning Research, 12, 2825-2830. 
Pennycook, G., \& Rand, D. G. (2019). Fighting Misinformation on Social Media Using Crowdsourced Judgments of News Source Quality. Proceedings of the National Academy of Sciences, 116(7), 2521-2526.

Pikulski, J. J. (2002). Readability. Boston: Houghton Mifflin.

Prasad, A. M., Iverson, L. R., \& Liaw, A. (2006). Newer Classification and Regression Tree Techniques: Bagging and Random Forests for Ecological Prediction. Ecosystems, 9(2), 181199.

Shearer, E. (2018). Social Media Putpaces Print Newspapers in the US as a News Source. Pew Research Center.

Siegel, A. A. (2019). Online Hate Speech. (accessed June 15, 2020; https://alexandrasiegel.com/wp-content/uploads/2019/08/Siegel_Online_Hate_Speech_v2.pdf).

Smith, D. N., \& Hanley, E. (2018). The Anger Games: Who Voted for Donald Trump in the 2016 Election, and Why? Critical Sociology, 44(2), 195-212.

Swibel, D. \& Boms, N. (2018). The Internet Hate Paradox. Israel Journal of Foreign Affairs, 12(1), 39-54.

Tibshirani, R. (1996). Regression shrinkage and selection via the lasso. Journal of the Royal Statistical Society: Series B (Methodological), 58(1), 267-288.

Trump Twitter Archive. (2020). Trump Twitter Archive. http://www.trumptwitterarchive.com/

Twitter. (2020). Twitter Safety on Twitter: “We added a label to two @realDonaldTrump Tweets about California's vote-by-mail plans as part of our efforts to enforce our civic integrity policy. We believe those Tweets could confuse voters about what they need to do to receive a ballot and participate in the election process." (Accessed May 27 https://twitter.com/twittersafety/status/1265838823663075341).

Valenzuela, S., Correa, T., \& Gil de Zuniga, H. (2018). Ties, Likes, and Tweets: Using Strong and Weak Ties to Explain Differences in Protest Participation across Facebook and Twitter Use. Political Communication, 35(1), 117-134.

Washington Post. (2020). Twitter Hides White House and Trump Minneapolis Tweet for 'Glorifying Violence. (Accessed July 4; https://www.washingtonpost.com/nation/2020/05/29/trumpminneapolis-twitter-protest/).

Wilson, T., Wiebe, J., \& Hoffmann, P. (2005). Recognizing Contextual Polarity in Phrase-Level Sentiment Analysis. Proceedings of Human Language Technology Conference and Conference on Empirical Methods in Natural Language Processing, 347-354.

Yang, H., Chan, L., \& King, I. (2002). Support vector machine regression for volatile stock market prediction. International Conference on Intelligent Data Engineering and Automated Learning, 391-396.

Yue, N. (2019). The "Weaponization" of Facebook in Myanmar: A Case for Corporate Criminal Liability Notes. Hastings Law Journal, 71(3), 813-844. 